\title{
Just a little magic
}

\section{Can magic tricks improve cooperation in strong-willed children?}

\begin{abstract}
Peretz B, Gluck G.
Magic trick: a behavioural strategy for the management of strongwilled children. Int J Paediatr Dent 2005; 15:429-436

Design This study was a randomised controlled trial.

Intervention Children identified as manifesting strong-willed behaviour (refusal to enter room and/ or sit in the dental chair) were selected and assigned, randomly, to test or control groups. Children in the test group ("Magic+") were shown a magic trick using a magic book (pictures erased magically then drawn again) prior to being encouraged to sit in the chair whereas those in the control group ("Magic-") were managed with 'tell-show-do' and positive reinforcement.
\end{abstract}

Outcome measure The behaviour of the two groups was assessed by measuring the time taken from the beginning of the session to the child sitting on the dental chair; whether radiographs were successfully taken; and using Frankl's behaviour category ${ }^{1}$, as rated by the dentist.

Results Thirty-five children, aged between 3 and 6 years, were allocated to each group. Time to sitting on the dental chair was significantly shorter in the Magic+ group ( $P$ 0.001) and radiographs could be taken in significantly more children (91\%; P 0.0013) than in the Magic-group (54\%). The Magic+ group demonstrated more cooperative behaviour as categorised by Frankl.

Conclusions This study demonstrates that a magic trick improved cooperation of strong-willed children by expediting the movement of the child into the dental chair and allowing the dentist to take radiographs more easily.

\section{Commentary}

This is a novel and interesting paper that reports a trial of an innovative intervention for managing challenging behaviour in the dental setting. The trial tests the effect of a specific intervention, the demonstration of a simple magic trick by the dentist, on the subsequent behaviour of 3-6-year old children who had previously been assessed as manifesting "strong-willed" behaviour. Children were considered to be strong-willed if they were noncompliant, stubborn, argumentative and defiant, expressing this by refusal to enter the operatory, refusal to open their mouth, or by pushing the dentist away. Once selected, children were randomly assigned to the group seeing the magic trick or to the control group (classical tell-show-do). Clearly, the study design did not enable either the dentist or the subjects to be blind to which intervention was used.

Three outcome measures were used, the time taken for the child to sit in dental chair, whether radiographs could be taken and a rating of their behaviour (Frankl's well-recognised scale). The group of children showed the magic trick performed better than controls for all outcome measures.

Unfortunately, this study has a number of limitations. The same magic trick was used for all study-group subjects and, although it is likely that the same dentist saw all patients, this is not clearly stated. This is important, because multiple operators might have introduced other variables and variations in the rating of behaviour. Furthermore, the rating of behaviour by the dentist who also delivered the intervention is a less than ideal design which could lead to bias. This could have been be avoided by having behaviour rated by independent observers. In addition, if rating is carried out by reviewing video recordings, this assessment could be blind. Operational limitations made such a design impractical in this case but, as a result, the differences in behaviour rating for both groups must be viewed with caution. Time taken to achieve the child sitting in the dental chair and ability to subsequently take radiographs would be less subject to bias although, again, it would have been more robust to have a separate operator, blinded to the behavioural intervention used, attempt to take the radiographs.

A useful additional finding was that all the children in the Magic+ group who had been assessed by their parents as uncooperative did not allow the dentist to take radiographs. This echoes previous studies that suggested parental predictions about behaviour at initial appointments are often accurate.

In summary, this investigation suggests that a magic trick may be a useful component in the introduction of strong-willed children to a dental clinical and radiographic examination. This may work as an effective ice-breaker, diverting the child's attention from the clinical aspects of the visit, make the dentist seem less threatening, and facilitate better communication between the child and dentist. Further carefully constructed trials should be conducted to confirm the value of this technique when compared with other more traditional approaches to managing challenging child behaviour in the dental setting.

\section{Practice point}

The study suggest that a magic trick may be useful addition in managing strong-willed children but further carefully constructed trials are needed.

\section{Stephen Fayle}

Leeds Dental Institute, Leeds, UK

1. FrankI SN, Shiere FR, Fogels HR. Should the parent remain with the child in the dental operatory? J Dent Child 1962; 2: 150-163.

Evidence-Based Dentistry (2006) 7, 76.

doi:10.1038/sj.ebd.6400431 